Ein vollstz̆ndiger oder gekürzter Abdruck der Referate 1st

\title{
Namenregister
}

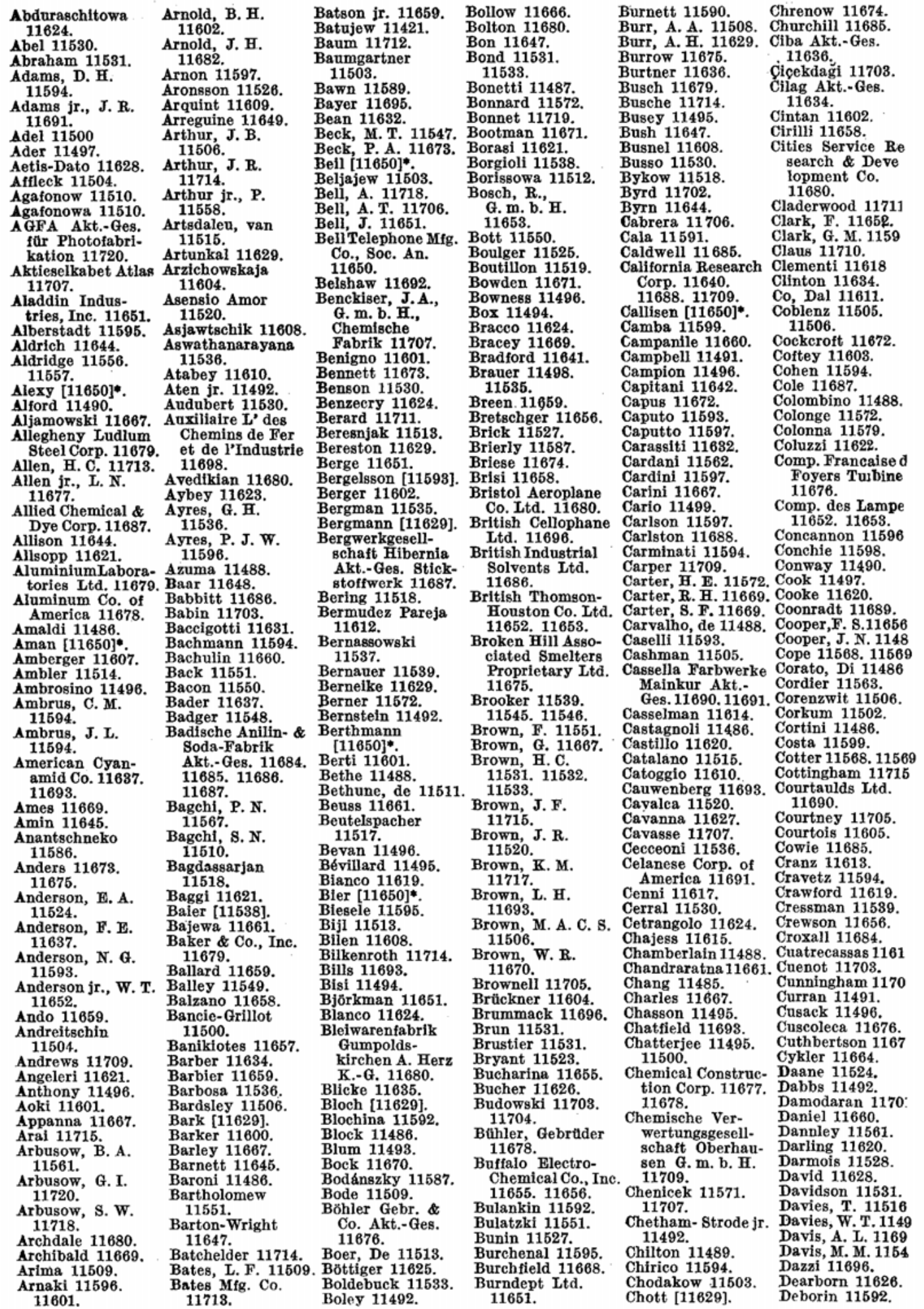




\section{CHEMISCHES ZENTRALBLATT}

VOLLSTÄNDIGES REPERTORIUM

FUR ALLE ZWEIGE DER REINEN UND ANGEWANDTEN CHEMIE

GEGRÜ N DET 1830

Im Auftrage der Deutsehon Akademie der Wissenschaften zu Berlin, der Chemischen Gesellschaft in der Deutsehen Demokratischen Republik, der Akademie der Wissenschaften zu Göttingen und der Gesellschaft Deutscher Chemiker

herausgegeben von

Prof. Dr. Maximilian Pfïcke

126. JAHRGANG

\section{5}

SEITE 10181-11749

Chefredakteure Dr. Eugen Klever und Prof. Dr. Maximilian Pfiucke 


\begin{tabular}{crr} 
Heft Nr. & Seitenzahl & \multicolumn{1}{c}{ Ausgabedatum } \\
\hline 44 & $10181-10424$ & 2. November 1955 \\
45 & $10425-10664$ & 9. November 1955 \\
46 & $10665-10904$ & 16. November 1955 \\
47 & $10905-11140$ & 23. November 1955 \\
48 & $11141-11320$ & 30. November 1955 \\
49 & $11321-11484$ & 7. Dezember 1955 \\
50 & $11485-11749$ & 14. Dezember 1955
\end{tabular}

Herausgeber und verantwortlich fur den Inhalt: Prof. Dr. Maximilian Pflacke, Berlin NW 7 , Schlffoauerdamm 19, Fernsprecher: 4255 71. Redalition: Chefredakteure Dr. Eu gen Klever u. Prof. Dr. Maximilian Pflucke. Veriag: Akademle-Verlag GmbH, Berlin W 8, MohrenstraBe 39 (Ferneprecher 200386, Postscheckkonto Berlln 35021), u. Verlag Chemie, GmbH., Weinheim/Bergstraße (Postscheckkonto Frankfurt/M. 145314 u. Berlln [West] 7430. Fernsprecher Weinhelm 2017). Bestell- und Verlags-Nr. 1007/126.

Satz u. Druck: VEB Lelpziger Druckhaus, Leipzig, III/18/203. I. 55.

Veróffentlicht unter der Llzenznummer 1212 des Amtes for Literatur u. Verlagswesen der Deutschen Demokratlschen Republik.

Printed in Germany. 


\title{
Bedarfsermittlung!
}

Der seit langer Zeit vergriffene zweite Quartalsband 1945 zum

\section{CHE'MISCHEN ZENTRALBLATT}

wird immer wieder wegen des darin befindlichen Autoren- und Patentregisters, 1. Halbjahr, verlangt.

Ein Nachdruck ist vorgesehen.

Bitte überprüfen Sie lhre Sammlung und geben Sie uns auf anhängender Karte Ihren Bedarf bekannt, damit eine genaue Auflage festgesetzt werden kann. Die Lieferung erfolgt gegebenenfalls nach Fertigstellung durch den Buchhandel.

\section{AKADEMIE - VERLAG GMBH Vertrieb}

\begin{abstract}
An den AKADEMIE-VERLAG, Vertrieb, Berlin W 8, Mohrenstraße 39
In meiner/unserer Sammlung des Chemischen Zentralblattes fehlt der zweite Quartalsband zum Jahrgang 1945.

Sọlte der Band nachgedruckt werden, benötige/n ich/wir Exemplare
\end{abstract}

zum Preise von DM 55, - und bitte um Lieferung durch die Buchhandlung

Bitte berücksichtigen Sie diese Anzahl bei der Festsetzung der Auflage Ort und Datum:

Name:

Anschrift: 
DRUCKSACHE

An den

Akademie-Verlag GmbH

BERLIN W 8

Mohrenstraße 39 
Dejak 11510. Ebrey 11547.

Demtschenko, L. G. Ediger, v. 11684. 11708.

Demtschenko, P. A. Ehrlich 11603. 11708.

Dennison 11490

Dent jr. 11539.

Deram 11579

Dessi 11614.

Destunis 11625

Deutsche Hydrierwerke Akt.-Ges. 11695.

Deutsche NortonGes. m. b. H. 11659.

Devonshire 11534.

Dhavalikar 11701.

Dichtjar 11702

Dickhăuser $[11650]^{*}$
Dickinson, D. G. 11628.

Dickinson, E. J. 11715.

Didier-Werke Akt.Ges. 11675 .

Diehl 11587.

Diemair [11650]*.

Dijkstra 11614.

Dilworth 11486 .

Dinsmore, H. L. 11549.

Dinsmore, R. P. 11698.

Distillere Co. Ltd. 11637.

Ehrhart [11650]*

Eidelman 11616 .

Eigen, H. 11658 .

Eigen, M. 11510 .

Eiland 11520.

clbe, v, 11516.

Electric \& Musica
Industries Ltd. Indust

Elektrokemisk A/S 11654

Fisher 11711

lliott, J. 11533. Fisk 11486.

Elliott, R. P. 11525. Fitzpatrlck 11626 soster Co.

11713

Ellison 11595

Elpiner 11592.

Elyash 11958.

Emich 11595.

England 11558.

Ensell 11495.

Flanders 11605.

Flaschka 11642.

Fleck jr. 11495.

Flick 11612 .

Flindt 11505 .

Florentin 11507.

Florian 11489.

Floyd 11685.

Foley 11489 .

Fonda 11652 .

ppelsheimer

11524.

Erb [11650]*.

11555 .

Erdmann-Jesnitz 11658.

Esdorn 11700

Esenwin 11658

Eser, G. 11596.

Eser, S. 11609. 11623.

Dmitrakowa 11508. Essig 11626.

Dodd 11657.

Dörling 11674.

Dogadkin 11698.

Dolecek 11506.

Dolega 11508.

Domagala 11526.

Donati 11612 .

Donnay, G. 11522

Donnay, J. D. H. 11522 .

Dorfman 11607.

Dorn 11653.

Dorschner 11682.

Dotti 11624

Douglas 11589

Etablissements Phillips \& Pain 11697.

Ethyl Corp. 11657.

Euler 11499.

Forrer 11506

Forsyth 11603.

Forziati 11591.

Foster 11683 .

Fourt 11711.

Fowden 11605 .

Fox 11659.

Franck 11512.

Frandsen 11535

Frank-Kamenetzki 11497.

Frankish 11683.

Franzinetti 11486 .

Freidlin 11551

Evans, H. D. 11596. Frensch [11650]*.

Evans, L. W. 11652. Frey [11629].

Evans, R. L. 11686. Freyer 11679.

Evans, T. 11719

Evenhuis 11706.

Everist 11706

Faessler 11498.

Fricke 11499

Friedenwald 11597.

Fritz 11646.

Froelich, H. C.

akstorp 11625 .

11653.

Falkenhagen 11510. Fröhlich, K. W.

Faragher 11699 [11650]*

11687 .

Drahowzal 11577.

Frolow 11592.

Drescher 11566 .

Farber 11704. Frost 11516. Akt.-Ges., vor- Fuchs [11650]*

11685 .

Drommond 11630. mals Meister Iucius Brüning 11687. 11712.

Dsbanowski 1158

Dubinin 11516 . 11517 .

Duff 11655.

Dumanski 11708.

Dunbar, D. N. F. 11489 .

Dunbar, S. R. 11524

Duncan, G. W. 11716 .

Duncan, L. K. 11534.

Dunlop, A. 11669.

Dunlop, A. K. 11552 .

Dunn 11494.

Du Pont de Nemours (E. I.) \& Co. 11656 . 11684. 11687. 11688. 11712

Dupuis 11615 .

Duran-Reynals 11593.

Dutta 11567.

Dworkin 11515.

Dyke, Van 11539.

Farhart 11694

Eastman Kodak 11685. 11718 . 11720 .

Ebeling 11508.

Ebert [11650]*
Farmer 11555.

Farney 11490.

Fullerton 11680

Farnsworth 11678. Fumetti, v. 11626.

Farren 11582

Farthing 11688 .

Faworskaja, N. A. 11582

Faworskaja, T. A. 11567.

Fazekas 11626.

Fedorenko, N. W. 11501.

Fedorenko, T. P. 11574.

Fedotow 11537.

Feinberg 11487.

Felice, de 11615 .

Fell 11621 .

Fellers 11705 .

Ferlin 11603.

Fernando, H. E. 11666.

Fernando, L. H 11661

11622.

Ferroni 14408.

. Feyfer, de 11492 .

Filosofo 11485 .

Finholt 11531.

11532. 11533 .
Felbert, v. [11650]"

Gerlach 11686.

Gersforif 11669 .

Gunn 11506 . $\quad 11654$.

Gussakowa 11582. Hersping 11508.

schinen Akt.-Ges. Gutzaljuk 11700. Heseltine 1154b.

11679 .

Gutzeit 11689.

Haaf 11626 .

Ghiringhelli 11621. Haas, F. L. 11602. Heyn 11671.

Haas, J. [11596]

Giannotti 11617. Haase 11649 .

Głbson 11506. Habōck 11659.

Giddey 11562

Gil Collado 11622. Hage [11650]*.

Hackmann 11.

Hageman 11597.

Gilbreath 11531. Hagemeyer jr. 11559 .

Gillespie 11704. Hahn 11490.

Giordani 11627. Haines 11638.

Giovannini 11704, Halama 11628.

Hall, A. N. 11602 .

Halliwell 11599.

Halpern 11491

Hamalainen 11711.

Hamill 11530 .

Hamilton 11489 .

Hammarlund

11631.

Gluesenkam

11695.

Godwin 11610.

Goehring 11535 .

Göttsche 11523.

Goey, de 11696 .

Gold farb 11573 .

Goldhaber, G.

11489.

Goldhaber, M.

11493.

Goldstein, $\mathrm{H}$.

11562.

Goldstein, M. S.

11596.

Gomberg 11705

Goodman 11626.

Goodrich, B. F.,

11681. 11686.

torbatschew 115

Hamme 11509.

Hammer 11713.

Hanemann 11672 .

Hannen 11647.

Hannewijk 11707.

Hanovia Chemical \& Mfg. Co. 11652

Hans-Heinrich11656.

Hanse 11702 .

Hansen 11526.

Hanson 11687.

Hantsch 11690.

Harbaugh 11678.

Hargreaves 11597. Hollaender 11700.

Gordon 11703.

Goritzkaja 11687

Gorjajew 11700

Goroshankina

11704.

Harris, G. M. 11495. Holm 11505.

Harris, J. C. 11489. Holmes, F. B.

Harrisson 11594.

Hartley 11670 .

Hartmann 11709.

Hartzler 11486 .

Gorra 11703. 11704. Harvey 11538.

Gorstein 11522 . 
Hyde 11531. 11532. Kamyschan 11708. Kolessnikow, N. Lejeune 11606. 11538. Kandiah 11669.

Hydrocarbon Re. Kantor 11618 .

search, Inc. $11656 \mathrm{~K}$ antyka 11655. 11489.11597.

Kolessnikow, N. N. Lembke 11600 11490.

11682. 11683.

Ijewinsch 11520 .

IIge 11653.

Imbo 11536 .

Imbriano 11604 .

Imelik 11552.

Imoto 11694.

Imperlal Chemical Industries Ltd.

11636. 11683

11685. 11686.

11688. 11691.

11692 .

Kapetanovic 11700. Komori 11609.

11681.

Kaplan 11552 .

Kapteyn 11651.

Karabinos 11603. Konkowa 11581.

Karapetjanz 11528. Konrad [11650]*

Karchmer 11643. 11683.

Kargin 11589.

Karischin 11574.

Karnofsky 11595 .

Karpow 11589.

Karthaus [11649].

Karunakar 11660 .

Imschenetzki 11663. Kassner 11509.

Ingenieur-Bureau Katritzky 11576.

Sonderegger \& Fi- Katz 11531. 11532.

scher 11675 .

Inghram 11644.

Ingold 11546 .

Ingraham 11485.

International Ge-

neral Electric

Co., Inc. 11656 .

Ioffe 11515 .

Iollewa 11518.

Kaufmann 11600 .

Kautter 11686 .

Kay, De 11630 .

Kayser 11682 .

Kedrow-Sichman

$$
11661 .
$$

Kedvessy 11632 .

Keenan 11497.

Ionics, Inc. 11697. Kegel 11674.

Ireland 11650.

Irving 11691.

Isaakidis 11666 . 11667.

Ishizawa 11663.

Istas 11647 .

Itoh 11547 .

Iwakiri 11662.

Iwanenko 11489 .

Iwanow 11534.

Iwtschenko 11706.

Jackson jr. 11561.

Jacob 11637.

Jacobsson 11612.

Jacox 11612 .

Jaffé 11703. 11704

Jakowlew 11567 .

Janensch 11702.

Jasper 11547.

Jatzuk 11705

Jayaraman 11499 .

11523.

Jedlícka 11651.

Jefremow 11614.

Jennings . 11711

Jensen, E. N.

11493.

Jensen, F. L. 11666.

Jensen, H. C. 11505

Jentoft 11640 .

Jentzer 11596.

Jeremitzkaja 11553.

Jesselsson 11513.

Jewell 11717.

Jillson 11524 .

Job 11632

Joerchel 11499.

Johansen 11534.

Johnson, F. D.

11693.

Johnson, F. H. [11596].

Johnson, G. R. A. 11646 .

Johnson, I. 11534.

Keitel 11703.

Kelbg 11510.

Korányi 11657.

Korenjako 11663

Korinth 11692 .

Kornberg 11599.

Korsching 11513

Korssakowa 11573

Kosmider 11676.

Kotani 11681.

Kowner 11548.

Kränzle 11629 .

Kraft, K, 11640 .

Krakoff 11595 .

Kramer 11596.

Kramers 11513

Krassilnikow 1

Kraybill 11706 .

Kriger 11537.

Krikorowa 11705.

Krippner 11630 .

Keller, F. 11523. Kristensen 11563.

Kelley 11715 .

Kritzkaja 11526 .

Kellogg, M. W., Co. Kronig 11514.

11650. 11683 .

11717.

Krutman 11559.

Kelly, P. 11550

Kelly, W. H. 11493. Krylatow 1170 11695.

Kennedy, w. K.

Kudrjawzew 11

Kuhnelt 11676 .

Kaley 11617 .

B . 11490, Kümmel 11487.

Kern, B. D. 11490 . Küntzel 11528 .

Kerr 11513.

Keste 11674.

Kuizenga 11638 .

Kuleschow 11527.

ettenbach 11640. Kumarnit 11720 .

Keyes 11539. 11545. Kunz 11686. 11546.

Keyl 11689.

Kichenassamy

11497.

Kinderman 1149

Kindschy 11715 .

King, D. T. 11486.

Kunze 11690.

Kupka 11615.

Kurbatov 11494.

Kurbatov 11494. Lopez 11705.

Kurt 11700 .

Kurti 11514.

Kuschnerew 11674

Kusstowa 11501 .

Kinnunen 11645. $\quad$ Kutepow 11685.

Kirab ya hi 11712. Labo 11814.

Lad 11531.

Lennert-Petersen 11603.

Lepore 11487.

Lepsius 11490.

Lessjuiss 11655 .

Letsinger 11563.

Lettré 11635.

Levin 11638. 11639.

Levine 11596.

Levinger 11525 .

Tis, W. E. 11493. Malinowski 11553.

Ley [11650]*.

i, Yin-Yuan 11520. Titanium Corp.

11538

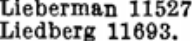

Liljegren 11658 .

Linch 11688.

Lindhard 11499.

Lindquist 11616 .

Linnell 11641.

Lipetz 11710 .

Little 11610 .

Livingston 11681.

Livrea 11611.

Loan 11590.

by 11509.

Lockwood 11694

Locsin 11660.

oebich 11672 .

oeffler, $\mathbf{F}$.

Lŏffler, J. 116

Lœuille 11641.

Loewe Opta Akt.Ges. 11651.

Lohss 11648.

Longini 11652.

Longwell 11516.

mische Fabriken Martin, P. K.1163.

Akt,-Ges, 11686, Martín García

Loon, van 11619.

11705.

Lorenz, W. 11658

Lossing 11546 .

Lota 11620.

Lounsbury 11551.

Low 11487.

Lozano Morales 11622.

Kirkpatrick 11648. Lafourcrière 11501. Lu 11493.

Kitaigorodski

Jones, J. I. 11590. Knoll Akt.-Ges.

Jones, R. V. 11716. Chemische Fa-

Joó 11604.

Joseph 11687.

Joshi 11577.

Juda 11697.

Julicher 11689.

Jung [11650]*

Jurkiewicz 11538 .

Juvet jr. 11529 .

Kaiser [11650]*.

Kallauner 11659 .

Kallee 11648 .

Kallenbach

[11650]*

Kaltofen 11714.

Kalvoda 11633.

Kamal 11613.

Kamlet 11656 . 11589.

Klages 11502.

Klaiber 11494 .

Kleckner 11633.

Kleffner 11654 .

Klein 11506.

Kleinsorg 11611.

Klimenkow 1

Knappwost 11620 .

Knight 11531.

briken 11640 .

Knoth jr. 11688 .

Knowles, M. B. 11718.

Knowles, W. S.

$$
11688 .
$$

Lagutotschkin

11663.

Lum 11688.

Lumalampan Ak

Lamanna $11693 . \quad$ tiebolag 11651.

Lamb 11677.

LaMoreaux 11538. Lundewall 11654.

Iang [11650]*

I ange, A. G.11630.

Lange, H. 11639. Lyons 11555

Langer, A. 11501. Lythgoe 11589.

Langer, E. [11629]. McAlpine 11646.

Lapilover 11620 .

Lapina 11617 .

Lapinskaja 11537.

Laplace 11624

Lare, Van 11539

Larin, Ss. 11489.

Larin, S8. J. 11

Laskowskt 11634.

Latzko 11607 .

Knulsli 11627.

Koch, J. [11650]*

Koch, L. 11674.

Todenko 11

Kölsch 11685 .

König 11696 .

Köszegi 11710.

Kofman 11578.

Kohan 11610.

Kolarow 11522 .

Lazar 11493.

Teandri 11561.

Lederer 11644
Laux [11650]* 
N.V. Industricele Ong, to 11665 . en Handelmij. Ongley 11646 . "Senzora" vhn. Oprischtschenko A.J. Schoemaker 11705. \& Sonen 11709 . Orr 11626 .

N. V. Philips' Gloci- Orrego Matte 11610. Pitler 11679. lampenfabrieken Osborne 11514. 11655. 11688. Oslage 11613.

Nadshakow 11504. Osol, A. 11633.

Nagai 11659.

Nagata 11609.

Naha 11500.

Nakagawa 11660.

Nakamura 11507. 11508.

Naoúm [11650]*.

Narlsada 11702

Nasarow 11583 .

11585. 11586. [11593].

Nasio 11628 .

Natali 11630.

Nathan 11638.

Nathans 11491

National Distille Products Corp. 11694.

National Research Councll 11649. ational Resear Development Corp. 11669.

Natterer 11626.

Naumann 11493.

Nebbia 11646.

Nehemias 11705.

Nelson, E. L. 11660. Parry 11643.

Nelson, J. F. 11699. Passynski 11592.

Nelson, R. N.11669. Patkul 11705.

Nessmejanow 11515. Patton 11625 .

Neudöriffer 11573. Paul 11690.

Neugebauer 11628. Paulin 11704.

Newberg 11688. Pawljutschenko

Newman 11490 .

Nex 11687

Nicander 11619.

Nicholls, G. W. 11669

Nicholls, R. W. 11499 .

Nichols 11609.

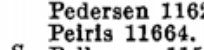

11650.

Nicholson, $\mathrm{M}$. 11527.

Nicol, A. D. I 11525 .

Nicol, T. 11608.

Nie, de 11700 .

Niggli 11536.

Nilsson 11498 .

Nöding 11622 .

Noll [11650]*.

Nomura 11503.

Nordal 11622.

Nordling 11645.

Norton 11525.

Nouvel 11634.

Nowacki 11522 .

Nurmikko 11600.

. Pellequer 11512.

. Petit 11535.

Odell 11552.

Oel- \& Chemic. Werk Akt.-Ges. 11675 .

Oelsen 11649

Onen 11629.

Oester 11619 .

Oesterreichisch-

Alpine Montan-

gesellschaft

11676 .

Ozkarakaya 11623. Petuely 11581.

Ogawa 11488.

Ogle 11720.

Ohlweiler 11646.

Ohno 11547.

Okada 11502.

Okonogi 11488.

Olivo 11614 .

O'Malley 11619 .

Oneda 11488 .

Raynolds 11695 .
Osol, J. K. 11520

Oswin 11696.

Ota 11694.

Otsu 11694.

Ott, A. C. 11637.

Ott, F. [11650]*

Ottaway 11611.

Otto, Georg 116

Otto, Gerhar

Oura 11498 .

Owen, J. 11509.

Owen, $\mathrm{T}$ 11554.

Owen, 0 11662. Poisson 11535.

Owens 11505. 11506. Poljakow 11714.

Pace 11650.

Pacheco 11606.

Paech 11607.

Pakschwer 11591. 11592.

11597.

Panetti 11487.

Pankow 11623.

Paolino 11596.

Parfitt 11673. 11674 11530.

Pearson, C. 11716 .

Pearson, J. 11669.

Peaslee 11490.

Peboc Ltd. 11682.

Pecsok 11529.

Pellini 11670.

Pena 11624.

Penati 11612.

Penner 11711.

Pepinsky 11520.

Perakis 11509.

Pereira Pinto 11606.

Peremans 11619.

Pereturina 11511.

Perry 11679

Peschkow 11514.

Peters 11630.

Petersky 11596.

Peterson, D. H. 11637.

Peterson, D. T. 11644.

Petot 11677

Petrenko 11537.

Petri [11650]*.

Petrocarbon Ltd. 11696. 11697.

Petrollte Corp. 11701.

Petrow 11552 11572.

Piirma 11491.

'iñus *1924.

ino 11622.

Pirona 11527.

Pittsburgh Plate

Glass Co. 11

11677.

Piwowarsky, K 11677.

czek 11519.

atzer 11685.

Plissow 11551.

Plöttner 11702.

oetz 11711.

odrizki 11613.

Pogni 11611.

Pohl 11485 .

Pohl-Rüling 11485 .

Pollock 11589.

Polonovski 1160

Pool 11492.

Poper 11696.

Popino 11632

Pouvreau 11536.

Powell 11680.

Powrie 11705.

Prader 11613.

Pratt 11558.

Preobrashenski

$$
11582 .
$$

Prettre 11519.

Prichard 11587.

Prigogine 11513.

Pringle 11494.

Britchard 11601.

Probst 11615.

Prodi 11521.

Prostenik 11572.

Prumbs 11666.

Puddington 11649. Roberts, L. D

Pylajew 11700 .

Quack [11650]*.

Quaker Oats Co.

11693.

Quehl 11712

Quittkat [1165

binowit

Rack 11682

Racker 11598 .

Rader 11645.

Radio Corp. of America 11678 .

Rae 11698 .

Räntzsch 11672. Rodrigo, D. M.

Rätze 11641.

Raffaele 11622.

Raffauf 11582 .

Raftery 11714 .

Raman 11499.

11523.

Rammler 11714.

Ramos 11708 .

Ramshorn 11648.

Rânby 11709.

Rao 11708.

Rapp 11531. 11532.

Rasskin 11664.

Raup 11661.

Rea 11626. Roquet 11507.

Read, T. A. 11527. Rose, K. 11670

Redden 11679 .

Reeve 11671 .

Regents of the University of Michi- 11709 .

gan 11635 .

Pfeifer 11508.

Pflanz 11625.

Philippot 11513.

Rehfeld 11630

Reich, F. 11527.

Co. 11683. 1169 $11700,11716$.

Pickholz 11682

Pleper 11673.

Pierri 11596.

Reimann 11613

Remes 11563 .

Rengarten 11537.

enner

Renold 11713

Reppe 11685.

Resegotti 11596.

Reuter 11587.

vol 11634.

nolds 11409.

Richardson, A.

ichardson, J. M. 11513.

Richardson, R. P.

11634

ter, F. 11674

Riede1, H. C. 11714 .

Riedel, W. 11714.

11564,11555 .

11566 .

Riester 11720 .

ndi 11599.

1687.

Risch 11630 .

itter, D. M. 11531 .

ivera Brenes 11706.

Rivero Arrarte

1610.

11609

Roberts, E. 1 11677.

rts, J. G. 11492.

rtson, J. H. 11644.

Robertson, W. D

Roblete 11670.

11523.

Robinson, R. 11 11634.

inson, R. J. 11640. 11662.

Rodrigo, F. A. 
Siedler [11650]*.
Siegel 11712.
Siemens \& Halske
Akt.-Ges. 11653.
11654.

Signer 11695.

Silbereisen 11672

Silvestroni 1161

Simone 11632.

Singer, S. E. 11494.

Singer, S. S.

11516.

Singh 11701.

Sirianni 11649.

Sitte 11485.

Sivertz 11551.

Skiles 11684.

Skoog 11563.

Slomp jr. 11638.

Smali 11663

Smedley 11693.

Smlrnowa 11665.

Smith, C. F. 11675.

Smith, H. G.

11524

Smith, H. N.

11521 .

Smith, J. E.

11486.

Smith jr., J. M. 11580 .

Smock 11663.

Smoluchowski

11520.

Snell 11608.

Snyder 11669.

Sô 11609 .

Soc. An. des Manufactures des

Glaces et Pro-

duits Chimiques

de Saint-Gobain,

Chauny \& Cirey

11697.

Soc. à r. 1. Vibur 11702.

Soc. Dorig-Girod 11680

Soc. a'Électro-

Chimie d'Electro

Metallurgie et des

Aciéries Êlec-

triques d'Ugine

11656.

Soc. Nobel Francaise, Soc. An. 11713 .

Soc. des Usines 11621 . Poulenc 11637. Strunz 11648.

Socony-Vacuum Oil Stubbs 11664.

Co., Inc. 11689. Sugayana 11702.

Sörensen 11641.

Sola 11626.

Solari 11624.

Solis 11718.

Solowjeff 11673 .

Sommers 11580.

Sookne 11711.

Soto del Basil 11610.

Soto-Rivera 11 Spänig 11686.

-Spahrkäs 11528.

Spauszus 11668 .

Spedding 11524 .

Spencer 11525 .

Spero 11638.

11639.

Sperry Corp.

11720.

Spisni 11606.

11622.

Sprague 11539.

Sprungnoli 11611.

Srinivasan 11530 .

Ssaatschan 11704.

Ssalganik 11618 .

ssonow

Sillantjewa 11522

suchow 11664 .

11666.

Stals 11696.

Development

Co. 11650 .

11681. 11683 .

11684. 11688 .

5. 11699.

11716. 11717.

Stanolind Oil and

Gas Co. 11650.

1685

Starr, M. P. 11705.

Starr, P. B. 11705.

tary 11596.

11601

tauss 11508

Staveley 11516.

11709.

teadman 11681.

Stefano, di 11623.

Stehlik 11521

Steiner 11696 .

Steinwehr, v.

11535. 11537 .

Steipe 11509.

telletzkaja 11526.

Stelmachow

11705.

tephenson 11492.

terling Drug,

Inc. 11634.

. 11598.

Stevens 11509

Stevenson 11501.

Stewart 11663

Stjaschkina 11708 .

Stöa 11622.

tone 11602.

Storonkin 11528.

Storrs 11668 .

Strain 11492

Strashessko 11518 .

Straub 11630.

Strauch 11488.

Streicher 11679 .

Stresen-Reuter,

Fred'k A., Inc. 11689 .

Sukacev 11651.

Suter 11688 .

Sutra 11528.

Suzuki, H. 11702 .

Suzuki, T. 11660.

Swan 11550.

Swann 11502.

Swazy 11679 .

Swell 11612.

Swerschkow

11621

Sweschnikow

11661.

Swim 11514.

Swiss 11626.

Sykes, M. P.

11595 .

Sykes, W. 11716.

Sylvania Electric

Products, Inc.

11652.

Symonds 11671. 11675.

Szekely 11630 .

Szepesy 11632 .

Szilárd 11604.

Ssamollowa 11561. Szwarc 11696 .
Tabor 11527.

Takaki 11507.

11508.

Takeda 11660

Taketomi 11702 .

Talbot 11619 .

Talboy jr. 11493.

Talenti 11538.

Tamele 11673 .

Tamini 11624.

Tan 11595.

Tanaka 11702 .

Tapavicza, v.

11623.

Tar, de 11662 .

Tarnóczy 11604 .

Tarnopolsky

1610.

rtakowskaja

11518

. 11673.

11500.

aylor, E. S.

11626.

Taylor, G. W. 11680 .

Taylor, H. W.

11494

Taylor, R. G. 11601.

Teague 11534.

Tebble 11507.

Technical Assets, Inc. 11684.

Teeter 11692 .

Terentjew 11582.

Terjajewa 11660.

Terry 11619 .

Texas Co. 11656. 11718.

Težak 11529 .

Theil 11707.

Thellung 11514 .

Thénard 11497.

Thews 11670.

Thibault 11701.

Thinius [11650]*.

Thirring 11488.

Thompson, H.

11691.

Thompson, L. M. 11552.

Thompson, Ralph B. 11570 . 11696.

Thorn 11488.

Thornton 11663.

Tihen 11715 .

Tilton 11644.

Tinlot 11485 .

Tischer 11705 .

Tissières 11596 .

Titus 11488.

Tjerkstra 11513.

Tjulin 11661.

Tjutin 11507.

Todd 11589 .

Toland 11688.

Tolnoek 11512.

Toman 11516.

Tomasini 11487 .

Tomic 11538.

Tomícek 11631.

Tomiser 11499.

Tompkins 11489.

Torgow 11583 .

11585. 11586.

Tosstanowskaja

11621.

Tournaire 11671.

Townes [11500].

Trambouze 11552 .

Trantina 11710 .

Travis 11537.

Trecco, de 11649

Triem 11686.

Trieschmann

11687.

Trippett 11687 .

Trocmé 11659.

Trotter 11620.

Tschernobilska 11663.

Tschernow 11660. Vittum 11720.

Tschessalin 11664. Vivian 11657

Tschetwerikowa Vimmeren, van 11604.

Tschirkow 11529. Völk 11662

Tschugunow

11571.

Tschujenkow

11548.

Tsubokawa 11523.

Tsujimura 11681 .

Tuchlow 11592 .

Tuck 11531.

11533.

Türcke 11674.

Tundo 11561.

Tung-Sol Iamp

Works, Inc.

11694.

Turchinetz 1149

Turhan 11615 .

Turkeltaub 116

Turner 11549.

Tusa 11685 .

Twerdowski

11515. 11517.

Tyler 11560.

Uchino 11605 .

Udy 11525 .

Ulagay 11611

Ullyot 11582.

Ulmanis 11507.

Ulrich 11704. 